\title{
Extremal dependence: some contributions
}

\author{
Helena Ferreira Department of Mathematics, University of Beira Interior, Covilhã, Portugal \\ Marta Ferreira Department of Mathematics, University of Minho, Braga, Portugal
}

\begin{abstract}
Due to globalization and relaxed market regulation, we have assisted to an increasing of extremal dependence in international markets. As a consequence, several measures of tail dependence have been stated in literature in recent years, based on multivariate extreme-value theory. In this paper we present a tail dependence function and an extremal coefficient of dependence between two random vectors that extend existing ones. We shall see that in weakening the usual required dependence allows to assess the amount of dependence in $d$-variate random vectors based on bidimensional techniques. Very simple estimators will be stated and can be applied to the well-known stable tail dependence function. Asymptotic normality and strong consistency will be derived too. An application to financial markets will be presented at the end.
\end{abstract}

Keywords: multivariate extreme value theory, tail dependence, extremal coefficients

\section{Introduction}

Dependence between extremal events have increased in recent time periods in financial markets, especially during bear markets and market crashes. The globalization and the lack of supervision are well-known contributions for this phenomena. Therefore, modern risk management is highly interested in assessing the amount of extremal dependence. The concept of tail dependence is the current tool used to this end, although it was first introduced far back in the sixties (Sibuya [25], 1960; Tiago de Oliveira [26], 1962/63). Tail dependence coefficients measure the probability of occurring extreme values for one random variable (r.v.) given that another assumes an extreme value too. These coefficients can be defined via copulas of random vectors which refers to their dependence structure concerning extreme events independently of their marginal distributions. The tail dependence coefficient,

$$
\lambda=\lim _{t \downarrow 0} P\left(F_{X}(X)>1-t \mid F_{Y}(Y)>1-t\right)
$$

where $F_{X}$ and $F_{Y}$ are the distribution functions (d.f.'s) of $X$ and $Y$, respectively, is perhaps the most referred in literature and characterizes the dependence in the tail of a random pair $(X, Y)$, i.e., $\lambda>0$ corresponds to tail dependence and $\lambda=0$ means tail independence. There are several references on this topic (besides the two above) and thus we point out only some of them: Ledford and Tawn (12, 13, 1996, 1997), Joe ([10, 1997), Coles et al. ([2], 1999), Embrechts et al. ([5], 2003).

Multivariate formulations for tail dependence coefficients can be used to describe the amount of dependence in the orthant tail of a multivariate distribution (Marshall-Olkin [17] 1967; Wolff 27] 1980; Nelsen [18] 1996; Schmid and Schmidt [21] 2007; Li [14, 15, 16] 2006, 2008, 2009, among others). These have been increasingly used in the most recent and higher demanding times. Most of the multivariate measures consider that extremal events must occur to all the components of the random vector, and obviously they are more complicated to deal with and to understand than in the bivariate case. Not surprisingly, applications hardly go any further than the three-dimensional case.

But maybe this is a too demanding condition and the occurrence of at least one extremal event in sub-vectors of a random vector can be enough to assess dependence. As already mentioned, 
financial markets are increasingly connected and the occurrence of at least one market crash, for instance, in Europe, will certainly influence a negative behavior in USA markets.

Based on this, we define a new tail dependence function for a random vector as a measure of the probability of occurring extreme values for the maximum of one sub-vector given that the maximum of another assumes an extreme value too. At the unit point, this function gives rise to the here called extremal coefficient of dependence as it relates to the well-known extremal coefficient (Tiago de Oliveira 1962-63, Smith 1990). These extend, respectively, the concept of upper tail dependence function and upper tail dependence coefficient already stated in literature (see Schmidt and Stadtmüller (2006) and references therein). In deriving the moments of the random variables involved in this approach, we find very simple estimators that can be also applied to the well-known stable tail dependence function (for a survey on this function see e.g. Beirlant et al. [1, 2004). Asymptotic normality and strong consistency are proved.

This paper is organized as follows. In Section 2 we define our new upper-tail dependence function and the extremal coefficient of dependence. We present some properties and examples. We also analyze the case of asymptotic independence. In Section 3 we present estimators and derive the respective properties of asymptotic normality and strong consistency. Section 4 illustrates our approach through an application to financial data.

\section{Extremal dependence between two random vectors}

Let $\mathbf{X}=\left(X_{1}, \ldots, X_{d}\right)$ be a random vector with d.f. $F$ and continuous marginal d.f.'s $F_{i}$. For $I \subset\{1, \ldots, d\}$, define $M(I)=\bigvee_{i \in I} F_{i}\left(X_{i}\right)$ and $\mathbf{X}_{I}$ the sub-vector of $\mathbf{X}$ having r.v.'s with indexes in $I$. Consider $C_{F}$ the copula function of $F$, i.e.,

$$
F\left(x_{1}, \ldots, x_{d}\right)=C_{F}\left(F_{1}\left(x_{1}\right), \ldots, F_{d}\left(x_{d}\right)\right), \quad\left(x_{1}, \ldots, x_{d}\right) \in \mathbb{R}^{d} .
$$

We are going to study the dependence between extremal events concerning two sub-vectors, $\mathbf{X}_{I_{1}}$ and $\mathbf{X}_{I_{2}}$, where $I_{1}$ and $I_{2}$ are disjoint subsets of $\{1, \ldots, d\}$.

We start by extending in Definition 2.2 the concept of upper tail dependence function (see Schmidt and Stadtmüller (2006) and references therein) and from this we define a new tail dependence coefficient between two random vectors.

Definition 2.1. Let $I_{1}$ and $I_{2}$ be two non-empty subsets of $\{1, \ldots, d\}$. The upper-tail dependence function of $\mathbf{X}_{I_{1}}$ given $\mathbf{X}_{I_{2}}$ is defined as, for $(x, y) \in(0, \infty)^{2}$,

$$
\Lambda_{U}^{\left(I_{1} \mid I_{2}\right)}(x, y)=\lim _{t \rightarrow \infty} P\left(M\left(I_{1}\right)>1-\frac{x}{t} \mid M\left(I_{2}\right)>1-\frac{y}{t}\right),
$$

provided the limit exists.

By taking $x=y=1$, we have

$$
\Lambda_{U}^{\left(I_{1} \mid I_{2}\right)}(1,1)=\lim _{t \rightarrow \infty} P\left(M\left(I_{1}\right)>1-\frac{1}{t} \mid M\left(I_{2}\right)>1-\frac{1}{t}\right),
$$

which is a tail dependence coefficient greater than the one considered in Li and Sun (2008),

$$
\gamma=\lim _{t \rightarrow \infty} P\left(\bigcap_{i \in I_{1}} F_{i}\left(X_{i}\right)>1-\frac{1}{t} \mid \bigcup_{i \in I_{2}} F_{i}\left(X_{i}\right)>1-\frac{1}{t}\right),
$$

which in turn is greater than the coefficient of $\operatorname{Li}(2009)$ for $I_{1}=\{1, \ldots, d\}-I_{2}$,

$$
\tau=\lim _{t \rightarrow \infty} P\left(\bigcap_{i \in I_{1}} F_{i}\left(X_{i}\right)>1-\frac{1}{t} \mid \bigcap_{i \in I_{2}} F_{i}\left(X_{i}\right)>1-\frac{1}{t}\right) .
$$


The tail dependence coefficient $\Lambda_{U}^{\left(I_{1} \mid I_{2}\right)}(1,1)$ give us information about the probability of occurring some extreme value in $\left\{F_{i}\left(X_{i}\right), i \in I_{1}\right\}$ given that some extreme value occurs in $\left\{F_{i}\left(X_{i}\right), i \in\right.$ $\left.I_{2}\right\}$.

Before presenting the properties of function $\Lambda_{U}^{\left(I_{1} \mid I_{2}\right)}(x, y)$ that will be the basis for the definition of our coefficient, consider the following notation:

for $(x, y) \in(0, \infty)^{2}, \emptyset \subseteq I_{1}, I_{2} \subseteq\{1, \ldots, d\}$ and $i \in\{1, \ldots, d\}$, let

$$
a_{i}^{\left(I_{1}, I_{2}\right)}(x, y)=x \mathbf{1}_{I_{1}}(i)+y \mathbf{1}_{I_{2}}(i)+\infty \mathbf{1}_{\overline{I_{1} \cup I_{2}}}(i),
$$

where $\mathbf{1}(\cdot)$ is the indicator function, and

$$
l^{\left(I_{1}, I_{2}\right)}(x, y)=-\log F\left(a_{1}^{\left(I_{1}, I_{2}\right)}(x, y), \ldots, a_{d}^{\left(I_{1}, I_{2}\right)}(x, y)\right),
$$

with the convention that, when some of the arguments of $F$ are $\infty$ we understand the limit of $F$ as those arguments tend to $\infty$.

If $F$ is a multivariate extreme value distribution (MEV) with unit Fréchet marginals, we have

$$
l^{\left(I_{1}, I_{2}\right)}\left(x^{-1}, x^{-1}\right)=-\log (\exp (-x))^{\epsilon_{I_{1} \cup I_{2}}}=x \epsilon_{I_{1} \cup I_{2}},
$$

where $\epsilon_{I_{1} \cup I_{2}}$ is the extremal coefficient of $\mathbf{X}_{I_{1} \cup I_{2}}$ (Tiago de Oliveira 1962-63, Smith 1990).

Proposition 2.1. If $F$ is a MEV distribution with unit Fréchet marginals, then function $\Lambda_{U}^{\left(I_{1} \mid I_{2}\right)}(x, y)$ is defined and verifies

$$
\Lambda_{U}^{\left(I_{1} \mid I_{2}\right)}(x, y)=1+\frac{x \epsilon_{I_{1}}}{y \epsilon_{I_{2}}}-\frac{l^{\left(I_{1}, I_{2}\right)}\left(x^{-1}, y^{-1}\right)}{y \epsilon_{I_{2}}}
$$

Dem. We have

$$
\Lambda_{U}^{\left(I_{1} \mid I_{2}\right)}(x, y)=\lim _{t \rightarrow \infty} 1+\frac{1-P\left(M\left(I_{1}\right) \leq 1-\frac{x}{t}\right)}{1-P\left(M\left(I_{2}\right) \leq 1-\frac{y}{t}\right)}-\frac{1-P\left(M\left(I_{1}\right) \leq 1-\frac{x}{t}, M\left(I_{2}\right) \leq 1-\frac{y}{t}\right)}{1-P\left(M\left(I_{2}\right) \leq 1-\frac{y}{t}\right)} .
$$

On the other hand, for $\alpha_{i}^{\left(I_{1}, I_{2}\right)}(u, v)=u \mathbf{1}_{I_{1}}(i)+v \mathbf{1}_{I_{2}}(i)+\mathbf{1}_{\overline{I_{1} \cup I_{2}}}(i)$, it holds

$$
\begin{aligned}
& \lim _{t \rightarrow \infty}-t \log P\left(M\left(I_{1}\right) \leq 1-\frac{x}{t}, M\left(I_{2}\right) \leq 1-\frac{y}{t}\right) \\
= & \lim _{t \rightarrow \infty}-t \log C_{F}\left(\alpha_{1}^{\left(I_{1}, I_{2}\right)}\left(1-\frac{x}{t}, 1-\frac{y}{t}\right), \ldots, \alpha_{d}^{\left(I_{1}, I_{2}\right)}\left(1-\frac{x}{t}, 1-\frac{y}{t}\right)\right) \\
= & \lim _{t \rightarrow \infty}-\log C_{F}\left(\alpha_{1}^{\left(I_{1}, I_{2}\right)}\left(\left(1-\frac{x}{t}\right)^{t},\left(1-\frac{y}{t}\right)^{t}\right), \ldots, \alpha_{d}^{\left(I_{1}, I_{2}\right)}\left(\left(1-\frac{x}{t}\right)^{t},\left(1-\frac{y}{t}\right)^{t}\right)\right) \\
= & -\log C_{F}\left(\alpha_{1}^{\left(I_{1}, I_{2}\right)}(\exp (-x), \exp (-y)), \ldots, \alpha_{d}^{\left(I_{1}, I_{2}\right)}(\exp (-x), \exp (-y))\right) \\
= & -\log F\left(a_{1}^{\left(I_{1}, I_{2}\right)}\left(x^{-1}, y^{-1}\right), \ldots, a_{d}^{\left(I_{1}, I_{2}\right)}\left(x^{-1}, y^{-1}\right)\right) \\
= & l^{\left(I_{1}, I_{2}\right)}\left(x^{-1}, y^{-1}\right) .
\end{aligned}
$$

Therefore, dividing the numerator and denominator of the fractions in (11) by $t$, we obtain

$$
\begin{aligned}
\Lambda_{U}^{\left(I_{1} \mid I_{2}\right)}(x, y) & =1+\frac{l^{\left(I_{1}, \emptyset\right)}\left(x^{-1}, x^{-1}\right)}{l^{\left(\emptyset, I_{2}\right)}\left(y^{-1}, y^{-1}\right)}-\frac{l^{\left(I_{1}, I_{2}\right)}\left(x^{-1}, y^{-1}\right)}{l^{\left(\emptyset, I_{2}\right)}\left(y^{-1}, y^{-1}\right)} \\
& =1+\frac{-\log (\exp (-x))^{\epsilon_{1}}}{-\log (\exp (-y))^{\epsilon_{2}}}-\frac{l^{\left(I_{1}, I_{2}\right)}\left(x^{-1}, y^{-1}\right)}{-\log (\exp (-y))^{\epsilon_{I_{2}}}}
\end{aligned}
$$


Therefore, under the conditions of Proposition 2.1, we have

$$
y \epsilon_{I_{2}} \Lambda_{U}^{\left(I_{1} \mid I_{2}\right)}(x, y)=x \epsilon_{I_{1}} \Lambda_{U}^{\left(I_{2} \mid I_{1}\right)}(y, x)=x \epsilon_{I_{1}}+y \epsilon_{I_{2}}-l^{\left(I_{1}, I_{2}\right)}\left(x^{-1}, y^{-1}\right)
$$

and we will denote this common value as $\Lambda_{U}^{\left(I_{1}, I_{2}\right)}(x, y)$.

Definition 2.2. The upper-tail dependence function for random vector $\left(\mathbf{X}_{I_{1}}, \mathbf{X}_{I_{2}}\right)$ with d.f. $M E V$ and unit Fréchet marginals is defined as

$$
\Lambda_{U}^{\left(I_{1}, I_{2}\right)}(x, y)=x \epsilon_{I_{1}}+y \epsilon_{I_{2}}-l^{\left(I_{1}, I_{2}\right)}\left(x^{-1}, y^{-1}\right)
$$

and the extremal coefficient of dependence between $\mathbf{X}_{I_{1}}$ and $\mathbf{X}_{I_{2}}$ is given by $\Lambda_{U}^{\left(I_{1}, I_{2}\right)}(1,1)$, which we denote $\epsilon_{\left(I_{1}, I_{2}\right)}$ and hence

$$
\epsilon_{\left(I_{1}, I_{2}\right)}=\epsilon_{I_{1}}+\epsilon_{I_{2}}-\epsilon_{I_{1} \cup I_{2}} .
$$

The upper-tail dependence function (14) generalizes the relation of Huang (1992) corresponding to $I_{1}=\{1\}$ and $I_{2}=\{2\}$,

$$
\Lambda_{U}(x, y)=x+y-l_{\left(F_{1}\left(X_{1}\right), F_{2}\left(X_{2}\right)\right)}^{H}(x, y),
$$

where the stable tail dependence function in the right-side is given by

$$
l_{\left(F_{1}\left(X_{1}\right), F_{2}\left(X_{2}\right)\right)}^{H}(x, y)=\lim _{t \rightarrow \infty} t P\left(F_{1}\left(X_{1}\right)>1-\frac{x}{t} \vee F_{2}\left(X_{2}\right)>1-\frac{y}{t}\right) .
$$

Observe that by (11) we also obtain

$$
l^{\left(I_{1}, I_{2}\right)}\left(x^{-1}, y^{-1}\right)=\lim _{t \rightarrow \infty} t P\left(M\left(I_{1}\right)>1-\frac{x}{t} \vee M\left(I_{2}\right)>1-\frac{y}{t}\right)=l_{\left(M\left(I_{1}\right), M\left(I_{2}\right)\right)}^{H}(x, y) .
$$

Moreover, the upper-tail dependence function in (14) can be can be viewed as an extension of the bivariate upper-tail dependence function of Schmidt and Stadtmüller ([22], 2006), defined as

$$
\Lambda_{\left(F_{1}\left(X_{1}\right), F_{2}\left(X_{2}\right)\right)}^{S}(x, y)=\lim _{t \rightarrow \infty} t P\left(F_{1}\left(X_{1}\right)>1-\frac{x}{t}, F_{2}\left(X_{2}\right)>1-\frac{y}{t}\right),
$$

by taking in this limit the random pair $\left(M\left(I_{1}\right), M\left(I_{2}\right)\right)$ instead of $\left(F\left(X_{1}\right), F\left(X_{2}\right)\right)$. At the unit vector, the Schmidt and Stadtmüller upper-tail dependence function corresponds to the tail dependence coefficient $\lambda$ in (11), i.e., $\lambda=\Lambda_{\left(F_{1}\left(X_{1}\right), F_{2}\left(X_{2}\right)\right)}^{S}(1,1)$.

In the following, we present the expression of the tail-dependence function $\Lambda_{U}^{\left(I_{1}, I_{2}\right)}(x, y)$ and the value of the corresponding extremal coefficient $\epsilon_{\left(I_{1}, I_{2}\right)}$ for a $d$-variate random vector $\mathbf{X}$ with well-known distribution functions for its margins.

Example 2.1. Consider vector $\mathbf{X}$ with unit Fréchet margins and copula function $C_{\mathbf{X}}\left(u_{1}, \ldots, u_{d}\right)=$ $\prod_{l=1}^{\infty} \prod_{k=-\infty}^{\infty} u_{1}^{\alpha_{l k 1}} \wedge \ldots \wedge u_{d}^{\alpha_{l k d}}$, where $u_{j} \in[0,1], j=1, \ldots, d$, and $\left\{\alpha_{l k j},-\infty<k<\infty, 1 \leq j \leq\right.$ $d, l \geq 1\}$ is a family of non negative constants such that $\sum_{l=1}^{\infty} \sum_{k=-\infty}^{\infty} \alpha_{l k j}=1, j=1, \ldots, d$. The distribution of $\mathbf{X}$ is the $M E V$ marginal distribution of multivariate maxima of moving maxima processes considered in Smith and Weissman (24], 1996). We have

$$
l^{\left(I_{1}, I_{2}\right)}(x, y)=-\log C\left(e^{-a_{1}^{-1}(x, y)}, \ldots, e^{-a_{d}^{-1}(x, y)}\right)=\sum_{l=1}^{\infty} \sum_{k=-\infty}^{\infty} \bigvee_{j=1}^{d} a_{j}^{-1}(x, y) \alpha_{l k j} .
$$

Therefore,

$$
\begin{aligned}
& \Lambda_{U}^{\left(I_{1}, I_{2}\right)}(x, y)=l^{\left(I_{1}, \emptyset\right)}\left(x^{-1}, x^{-1}\right)+l^{\left(\emptyset, I_{2}\right)}\left(y^{-1}, y^{-1}\right)-l^{\left(I_{1}, I_{2}\right)}\left(x^{-1}, y^{-1}\right) \\
= & x \sum_{l=1}^{\infty} \sum_{k=-\infty}^{\infty} \bigvee_{j \in I_{1}} \alpha_{l k j}+y \sum_{l=1}^{\infty} \sum_{k=-\infty}^{\infty} \bigvee_{j \in I_{2}} \alpha_{l k j}-\sum_{l=1}^{\infty} \sum_{k=-\infty}^{\infty}\left(\left(x \bigvee_{j \in I_{1}} \alpha_{l k j}\right) \vee\left(y \bigvee_{j \in I_{2}} \alpha_{l k j}\right)\right)
\end{aligned}
$$


and

$$
\epsilon_{\left(I_{1}, I_{2}\right)}(x, y)=\sum_{l=1}^{\infty} \sum_{k=-\infty}^{\infty} \bigvee_{j \in I_{1}} \alpha_{l k j}+\sum_{l=1}^{\infty} \sum_{k=-\infty}^{\infty} \bigvee_{j \in I_{2}} \alpha_{l k j}-\sum_{l=1}^{\infty} \sum_{k=-\infty}^{\infty} \bigvee_{j \in I_{1} \cup I_{2}} \alpha_{l k j}
$$

Illustrating with

$C_{\mathbf{X}}\left(u_{1}, u_{2}, u_{3}, u_{4}\right)=\left(u_{1}^{1 / 8} \wedge u_{2}^{1 / 8} \wedge u_{3}^{1 / 8} \wedge u_{4}^{1 / 8}\right) \cdot\left(u_{1}^{5 / 8} \wedge u_{2}^{4 / 8} \wedge u_{3}^{7 / 8} \wedge u_{4}^{1 / 8}\right) \cdot\left(u_{1}^{1 / 8} \wedge u_{2}^{2 / 8}\right) \cdot\left(u_{1}^{1 / 8} \wedge u_{2}^{1 / 8} \wedge u_{4}^{6 / 8}\right)$,

$I_{1}=\{1,2\}$ and $I_{2}=\{3,4\}$, we obtain

$$
\begin{aligned}
\Lambda_{U}^{\left(I_{1}, I_{2}\right)}(x, y) & =\left(\frac{1}{8}+\frac{5}{8}+\frac{2}{8}+\frac{1}{8}\right) x+\left(\frac{1}{8}+\frac{7}{8}+\frac{6}{8}\right) y-\left(\left(x \frac{1}{8} \vee y \frac{1}{8}\right)+\left(x \frac{5}{8} \vee y \frac{7}{8}\right)+\left(x \frac{2}{8}\right)+\left(x \frac{1}{8} \vee y \frac{6}{8}\right)\right) \\
& =\frac{9}{8} x+\frac{14}{8} y-\left(\left(x \frac{1}{8} \vee y \frac{1}{8}\right)+\left(x \frac{5}{8} \vee y \frac{7}{8}\right)+x \frac{2}{8}+\left(x \frac{1}{8} \vee y \frac{6}{8}\right)\right)
\end{aligned}
$$

and

$$
\epsilon_{\left(I_{1}, I_{2}\right)}=\frac{9}{8}+\frac{14}{8}-\left(\frac{1}{8}+\frac{7}{8}+\frac{2}{8}+\frac{6}{8}\right)=\frac{7}{8}
$$

Similarly, if $I_{1}=\{1,2\}$ and $I_{2}=\{4\}$ we obtain

$$
\Lambda_{U}^{\left(I_{1}, I_{2}\right)}(x, y)=\frac{9}{8} x+y-\left(\left(x \frac{1}{8} \vee y \frac{1}{8}\right)+\left(x \frac{5}{8} \vee y \frac{1}{8}\right)+x \frac{2}{8}+\left(x \frac{1}{8} \vee y \frac{6}{8}\right)\right)
$$

and

$$
\epsilon_{\left(I_{1}, I_{2}\right)}=\frac{9}{8}+1-\left(\frac{1}{8}+\frac{5}{8}+\frac{2}{8}+\frac{6}{8}\right)=\frac{3}{8} .
$$

Example 2.2. For the symmetric logistic model we have

$$
l^{\left(I_{1}, I_{2}\right)}(x, y)=-\log F\left(a_{1}^{\left(I_{1}, I_{2}\right)}(x, y), \ldots, a_{d}^{\left(I_{1}, I_{2}\right)}(x, y)\right)=\left(\sum_{j=1}^{d}\left(a_{j}^{\left(I_{1}, I_{2}\right)}(x, y)\right)^{-1 / \theta}\right)^{\theta}
$$

with $\theta \in(0,1], x, y>0$. Therefore,

$$
\begin{aligned}
\Lambda_{U}^{\left(I_{1}, I_{2}\right)}(x, y) & =l^{\left(I_{1}, \emptyset\right)}\left(x^{-1}, x^{-1}\right)+l^{\left(\emptyset, I_{2}\right)}\left(y^{-1}, y^{-1}\right)-l^{\left(I_{1}, I_{2}\right)}\left(x^{-1}, y^{-1}\right) \\
& =\left(\sum_{j \in I_{1}} x^{1 / \theta}\right)^{\theta}+\left(\sum_{j \in I_{2}} y^{1 / \theta}\right)^{\theta}-\left(\sum_{j \in I_{1}} x^{1 / \theta}+\sum_{j \in I_{2}} y^{1 / \theta}\right)^{\theta} \\
& =\left|I_{1}\right|^{\theta} x+\left|I_{2}\right|^{\theta} y-\left(\left|I_{1}\right| x^{1 / \theta}+\left|I_{2}\right| y^{1 / \theta}\right)^{\theta}
\end{aligned}
$$

and

$$
\epsilon_{\left(I_{1}, I_{2}\right)}=\left|I_{1}\right|^{\theta}+\left|I_{2}\right|^{\theta}-\left(\left|I_{1}\right|+\left|I_{2}\right|\right)^{\theta}
$$

Proposition 2.2. Under the conditions of Proposition [2.1 we have

(i) $0 \leq \Lambda_{U}^{\left(I_{1}, I_{2}\right)}(x, y) \leq x \epsilon_{I_{1}} \wedge y \epsilon_{I_{2}}$

(ii) $0 \leq \epsilon_{\left(I_{1}, I_{2}\right)} \leq \epsilon_{I_{1}} \wedge \epsilon_{I_{2}}$.

\section{Dem.}

(i) The left inequality is straightforward by the definition of $\Lambda_{U}^{\left(I_{1}, I_{2}\right)}(x, y)$ in (14). Observe also that, since $\mathbf{X}$ has MEV distribution, it is associated (in the sense of Joe [10], 1997; Theorem 6.7) and hence, for all $I_{1}, I_{2} \subset\{1, \ldots, d\}$,

$$
\begin{aligned}
& F\left(a_{1}^{\left(I_{1}, I_{2}\right)}\left(x^{-1}, y^{-1}\right), \ldots, a_{d}^{\left(I_{1}, I_{2}\right)}\left(x^{-1}, y^{-1}\right)\right) \\
\geq & F\left(a_{1}^{\left(I_{1}, \emptyset\right)}\left(x^{-1}, x^{-1}\right), \ldots, a_{d}^{\left(I_{1}, \emptyset\right)}\left(x^{-1}, x^{-1}\right)\right) F\left(a_{1}^{\left(\emptyset, I_{2}\right)}\left(y^{-1}, y^{-1}\right), \ldots, a_{d}^{\left(\emptyset, I_{2}\right)}\left(y^{-1}, y^{-1}\right)\right),
\end{aligned}
$$


leading to the same conclusion, i.e.,

$$
l^{\left(I_{1}, \emptyset\right)}\left(x^{-1}, x^{-1}\right)+l^{\left(\emptyset, I_{2}\right)}\left(y^{-1}, y^{-1}\right)-l^{\left(I_{1}, I_{2}\right)}\left(x^{-1}, y^{-1}\right) \geq 0 .
$$

On the other hand,

$$
\begin{aligned}
& F\left(a_{1}^{\left(I_{1}, I_{2}\right)}\left(x^{-1}, y^{-1}\right), \ldots, a_{d}^{\left(I_{1}, I_{2}\right)}\left(x^{-1}, y^{-1}\right)\right) \\
\leq & F\left(a_{1}^{\left(I_{1}, \emptyset\right)}\left(x^{-1}, x^{-1}\right), \ldots, a_{d}^{\left(I_{1}, \emptyset\right)}\left(x^{-1}, x^{-1}\right)\right) \wedge F\left(a_{1}^{\left(\emptyset, I_{2}\right)}\left(y^{-1}, y^{-1}\right), \ldots, a_{d}^{\left(\emptyset, I_{2}\right)}\left(y^{-1}, y^{-1}\right)\right)
\end{aligned}
$$

and hence

$$
\begin{aligned}
& l^{\left(I_{1}, \emptyset\right)}\left(x^{-1}, x^{-1}\right)+l^{\left(\emptyset, I_{2}\right)}\left(y^{-1}, y^{-1}\right)-l^{\left(I_{1}, I_{2}\right)}\left(x^{-1}, y^{-1}\right) \\
\leq & l^{\left(I_{1}, \emptyset\right)}\left(x^{-1}, x^{-1}\right)+l^{\left(\emptyset, I_{2}\right)}\left(y^{-1}, y^{-1}\right)-\left(l^{\left(I_{1}, \emptyset\right)}\left(x^{-1}, x^{-1}\right) \vee l^{\left(\emptyset, I_{2}\right)}\left(y^{-1}, y^{-1}\right)\right) \\
= & x \epsilon_{I_{1}} \wedge y \epsilon_{I_{2}} . \square
\end{aligned}
$$

The result in (i) agrees with the one for the bivariate case. Observe that, from the proof above we can also conclude that the boundary cases correspond to, respectively, independence and total dependence.

Remark 2.1. With the conventions $1 / 0:=\infty$ and $1 / \infty:=0$, we can define $\Lambda_{U}^{\left(I_{1}, I_{2}\right)}(x, y)$ in $[0, \infty]^{2} \backslash\{(\infty, \infty)\}$ and found $\Lambda_{U}^{\left(I_{1}, I_{2}\right)}(0, y)=0=\Lambda_{U}^{\left(I_{1}, I_{2}\right)}(x, 0), \Lambda_{U}^{\left(I_{1}, I_{2}\right)}(\infty, y)=y \epsilon_{I_{2}}$ and $\Lambda_{U}^{\left(I_{1}, I_{2}\right)}(x, \infty)=$ $x \epsilon_{I_{1}}$.

Proposition 2.3. Under the conditions of Proposition 2.1 and Remark 2.1, for each $y \geq 0$, the partial derivative $\partial \Lambda_{U}^{\left(I_{1}, I_{2}\right)} / \partial x$ exists for almost all $x>0$, and

$$
0 \leq \frac{\partial}{\partial x} \Lambda_{U}^{\left(I_{1}, I_{2}\right)}(x, y) \leq\left|I_{1}\right|
$$

Similarly, for each $x \geq 0$, the partial derivative $\partial \Lambda_{U}^{\left(I_{1}, I_{2}\right)} / \partial x$ exists for almost all $y>0$, and

$$
0 \leq \frac{\partial}{\partial y} \Lambda_{U}^{\left(I_{1}, I_{2}\right)}(x, y) \leq\left|I_{2}\right|
$$

Also, the functions $x \mapsto \partial \Lambda_{U}^{\left(I_{1}, I_{2}\right)}(x, y) / \partial y$ and $y \mapsto \partial \Lambda_{U}^{\left(I_{1}, I_{2}\right)}(x, y) / \partial x$ are defined and non decreasing almost everywhere on $[0, \infty)$.

Dem. The function $\Lambda_{U}^{\left(I_{1}, I_{2}\right)}(x, y)$ is 2-increasing since a bivariate d.f. is 2-increasing. By Remark 2.1] we conclude that $\Lambda_{U}^{\left(I_{1}, I_{2}\right)}(x, y)$ is grounded. Hence, applying Lemma 2.1.5. in Nelsen $([19], 2006)$ we have, for $(x, y),\left(x^{*}, y^{*}\right) \in[0, \infty]^{2} \backslash\{(\infty, \infty)\}$,

$$
\begin{aligned}
& \left|\Lambda_{U}^{\left(I_{1}, I_{2}\right)}(x, y)-\Lambda_{U}^{\left(I_{1}, I_{2}\right)}\left(x^{*}, y^{*}\right)\right| \\
\leq & \lim _{t \rightarrow \infty} t\left(\left|P\left(M\left(I_{1}\right)>1-\frac{x}{t}\right)-P\left(M\left(I_{1}\right)>1-\frac{x^{*}}{t}\right)\right|+\left|P\left(M\left(I_{2}\right)>1-\frac{y}{t}\right)-P\left(M\left(I_{2}\right)>1-\frac{y^{*}}{t}\right)\right|\right) \\
\leq & \left|I_{1}\right|\left|x-x^{*}\right|+\left|I_{2}\right|\left|y-y^{*}\right| .
\end{aligned}
$$

Now, the proof is straightforward from Theorem 3 in Schmidt and Stadtmüller ([22], 2006).

Remark 2.1 and Propositions 2.2(i) and 2.3 extend, respectively, Theorems 1.i), 2.i) and 3 of Schmidt and Stadtmüller ([22, 2006). Moreover, given the above mentioned relation between $\Lambda_{U}^{\left(I_{1}, I_{2}\right)}(x, y)$ and the bivariate upper-tail dependence function $\Lambda_{\left(F_{1}\left(X_{1}\right), F_{2}\left(X_{2}\right)\right)}^{S}(x, y)$ in (19), the 
properties ii)-v) of Theorems 1 and 2 of Schmidt and Stadtmüller ([22], 2006) are straightforward for $\Lambda_{U}^{\left(I_{1}, I_{2}\right)}(x, y)$.

We now discuss the case of tail independence between $M\left(I_{1}\right)$ and $M\left(I_{2}\right)$ and hence extend our context beyond a MEV distribution.

Notice that, in case of tail dependence between r.v.'s $F_{1}\left(X_{1}\right)$ and $F_{2}\left(X_{2}\right)$, the mapping

$$
t \mapsto P\left(F_{1}\left(X_{1}\right)>1-\frac{x}{t}, F_{2}\left(X_{2}\right)>1-\frac{y}{t}\right)
$$

is regularly varying of order -1 at $\infty$, and so an homogeneity property holds for large $t$. However, if $\left(F_{1}\left(X_{1}\right), F_{2}\left(X_{2}\right)\right)$ is tail independent, this latter does not hold and an adjusted homogeneity property can be obtained by assuming that (20) is regularly varying of order $-1 / \eta$ at $\infty, \eta<1$ (the case $\eta=1$ corresponds to tail dependence). Coefficient $\eta$ is the coefficient of tail dependence introduced in Ledford and Tawn (1996, 1997).

Thus being, if we assume that (20) is regularly varying of order $-1 / \eta$ at $\infty$, i.e.,

$$
\lim _{t \rightarrow \infty} \frac{P\left(F_{1}\left(X_{1}\right)>1-x / t, F_{2}\left(X_{2}\right)>1-y / t\right)}{P\left(F_{1}\left(X_{1}\right)>1-1 / t, F_{2}\left(X_{2}\right)>1-1 / t\right)}=c^{*}(x, y)
$$

for $(x, y) \in[0, \infty)^{2}$, where $c^{*}$ is homogeneous of order $1 / \eta$ for some $\eta \in(0,1]$ and $c^{*}(1,1)=1$, then $t \mapsto P\left(F_{1}\left(X_{1}\right)>1-1 / t, F_{2}\left(X_{2}\right)>1-1 / t\right)$ is regularly varying at $\infty$ with index $-1 / \eta$ (choose $x=y$ in (21) , and hence we can write

$$
P\left(F_{1}\left(X_{1}\right)>1-1 / t, F_{2}\left(X_{2}\right)>1-1 / t\right)=t^{-1 / \eta} L(t)
$$

where $L$ is a slowly varying function at $\infty$ (i.e., $L(t x) / L(t) \rightarrow 1$, as $t \rightarrow \infty$, for any $x>0$ ). Observe that $\eta$ dominates the speed of convergence of $P\left(F_{1}\left(X_{1}\right)>1-1 / t, F_{2}\left(X_{2}\right)>1-1 / t\right)$ to 0 . If $\eta<1$ then $F_{1}\left(X_{1}\right)$ and $F_{2}\left(X_{2}\right)$ (and thus $X_{1}$ and $X_{2}$ ) are asymptotically independent (or tail independent). In this case, the tail dependence coefficient $\lambda$ in (1) is null. Conversely, asymptotic dependence holds if $\eta=1$ and $L(t) \rightarrow a>0$, as $t \rightarrow \infty$, and we have $\lambda>0$. If $\eta=1 / 2$ we have (almost) independence (perfect independence if $L(t)=1$ and (21) holds with $\left.c^{*}(x, y)=x y\right)$. The cases $\eta \in(0,1 / 2)$ and $\eta \in(1 / 2,1)$ correspond to asymptotically negative independence and to asymptotically positive independence, respectively. Roughly speaking, coefficient $\eta$ governs a kind of a pre-asymptotic tail behavior that allows to better estimate the probability of extreme events in case of tail independence. A bivariate extreme value distribution (BEV) allows only tail dependence $(\eta=1)$ or independence $(\eta=1 / 2)$, since

$$
P\left(F_{1}\left(X_{1}\right)>1-1 / t, F_{2}\left(X_{2}\right)>1-1 / t\right) \sim\left(2-l^{(\{1\},\{2\})}(1,1)\right) / t+\left(\left(l^{(\{1\},\{2\})}(1,1)\right)^{2} / 2-1\right) / t^{2}
$$

as $t \rightarrow \infty$. For a discussion on this topic see, for instance, Ledford and Tawn ([12], 1996), Draisma et al. $([3,2004)$ and Drees and Müller $([4,2008)$.

Now assume that (21) holds for random pair $\left(M\left(I_{1}\right), M\left(I_{2}\right)\right)$, i.e.,

$$
\lim _{t \rightarrow \infty} \frac{P\left(M\left(I_{1}\right)>1-x / t, M\left(I_{2}\right)>1-y / t\right)}{P\left(M\left(I_{1}\right)>1-1 / t, M\left(I_{2}\right)>1-1 / t\right)}=c_{\left(I_{1}, I_{2}\right)}(x, y)
$$

for $(x, y) \in[0, \infty)^{2}$, where $c_{\left(I_{1}, I_{2}\right)}$ is homogeneous of order $1 / \eta_{\left(I_{1}, I_{2}\right)}$ for some $\eta_{\left(I_{1}, I_{2}\right)} \in(0,1]$ and $c_{\left(I_{1}, I_{2}\right)}(1,1)=1$. Taking $x=y$ in (23), one obtains that $P\left(M\left(I_{1}\right)>1-1 / t, M\left(I_{2}\right)>1-1 / t\right)$ is regularly varying at $\infty$, i.e.,

$$
P\left(M\left(I_{1}\right)>1-1 / t, M\left(I_{2}\right)>1-1 / t\right)=t^{-1 / \eta_{\left(I_{1}, I_{2}\right)}} L_{\left(I_{1}, I_{2}\right)}(t),
$$


where $L_{\left(I_{1}, I_{2}\right)}(t)$ is a slowly varying function at $\infty$. Coefficient $\eta_{\left(I_{1}, I_{2}\right)}$ is now a measure of the speed of convergence of $P\left(M\left(I_{1}\right)>1-1 / t, M\left(I_{2}\right)>1-1 / t\right)$ to 0 and is, therefore, a coefficient of tail dependence between $M\left(I_{1}\right)$ and $M\left(I_{2}\right)$, with analogous conclusions derived for $\eta$ above. Similarly, in a MEV we obtain, as $t \rightarrow \infty$,

$$
P\left(M\left(I_{1}>1-1 / t, M\left(I_{2}\right)>1-1 / t\right) \sim\left(\epsilon_{I_{1}}+\epsilon_{I_{2}}-\epsilon_{I_{1} \cup I_{2}}\right) / t+\left(\epsilon_{I_{1} \cup I_{2}}^{2}-\epsilon_{I_{1}}^{2}-\epsilon_{I_{2}}^{2}\right) /\left(2 t^{2}\right) .\right.
$$

Hence it only occurs asymptotic dependence whenever $\epsilon_{\left(I_{1}, I_{2}\right)}=\epsilon_{I_{1}}+\epsilon_{I_{2}}-\epsilon_{I_{1} \cup I_{2}}>0$ (with $\left.\eta_{\left(I_{1}, I_{2}\right)}=1\right)$, and otherwise independence $\left(\eta_{\left(I_{1}, I_{2}\right)}=1 / 2\right)$.

In the next result we compute $\eta_{\left(I_{1}, I_{2}\right)}$ and found that it is given by the maximum coefficient $\eta_{\{i\},\{j\}}$, $\forall i \in I_{1}, j \in I_{2}$.

Proposition 2.4. Suppose that 24) holds and

$$
P\left(\min _{i \in I, j \in J}\left(F_{i}\left(X_{i}\right), F_{j}\left(X_{j}\right)\right)>1-1 / t\right)=t^{-1 / \eta_{I, J}} L_{\eta_{I, J}}(t)
$$

holds for all $\emptyset \neq I \subset I_{1}$ and $\emptyset \neq J \subset I_{2}$, where $L_{\eta_{I, J}}$ is a slowly varying function at $\infty$. Then $\eta_{\left(I_{1}, I_{2}\right)}=\max \left\{\eta_{\{i\},\{j\}}: i \in I_{1}, j \in I_{2}\right\}$.

Dem. First observe that if $I^{\prime} \subset I$ and $J^{\prime} \subset J$ then

$$
1 \geq t^{-1 / \eta_{I^{\prime}, J^{\prime}}} L_{\eta_{I^{\prime}, J^{\prime}}}(t) \geq t^{-1 / \eta_{I, J}} L_{\eta_{I, J}}(t) .
$$

We have that

$$
\begin{aligned}
& P\left(\bigvee_{i \in I_{1}} F_{i}\left(X_{i}\right)>1-1 / t, \bigvee_{j \in I_{2}} F_{j}\left(X_{j}\right)>1-1 / t\right) \\
= & P\left(\bigcup_{i \in I_{1}}\left\{F_{i}\left(X_{i}\right)>1-1 / t, \bigcup_{j \in I_{2}}\left\{F_{j}\left(X_{j}\right)>1-1 / t\right\}\right\}\right) \\
= & \sum_{\emptyset \neq S \subseteq I_{1}}(-1)^{|S|+1} P\left(\bigcap_{i \in S}\left\{F_{i}\left(X_{i}\right)>1-1 / t\right\}, \bigcup_{j \in I_{2}}\left\{F_{j}\left(X_{j}\right)>1-1 / t\right\}\right) \\
= & \sum_{\emptyset \neq S \subseteq I_{1}} \sum_{\emptyset \neq T \subseteq I_{2}}(-1)^{|S|+|T|} P\left(\bigcap_{i \in S}\left\{F_{i}\left(X_{i}\right)>1-1 / t\right\}, \bigcap_{j \in T}\left\{F_{j}\left(X_{j}\right)>1-1 / t\right\}\right) \\
= & \sum_{\emptyset \neq S \subseteq I_{1}} \sum_{\emptyset \neq T \subseteq I_{2}}(-1)^{|S|+|T|} t^{-1 / \eta_{S, T}} L_{\eta_{S, T}}(t),
\end{aligned}
$$

where in the last equality we have applied (25). Let

$$
\eta=\max _{\emptyset \neq S \subseteq I_{1} \emptyset \neq T \subseteq I_{2}} \eta_{S, T}
$$

From (27) and (28) we have that

$$
\begin{aligned}
& P\left(\bigvee_{i \in I_{1}} F_{i}\left(X_{i}\right)>1-1 / t, \bigvee_{j \in I_{2}} F_{j}\left(X_{j}\right)>1-1 / t\right) \\
= & t^{-1 / \eta} L_{\eta}(t) \sum_{\emptyset \neq S \subseteq I_{1}} \sum_{\emptyset \neq T \subseteq I_{2}}(-1)^{|S|+|T|} A_{S, T}(t)
\end{aligned}
$$

where $A_{S, T}(t)=t^{-\left(1 / \eta_{S, T}-1 / \eta\right)} L_{\eta_{S, T}}^{*}(t)$ and $L_{\eta_{S, T}}^{*}(t)=L_{\eta_{S, T}}(t) / L_{\eta}(t)$ is a slowly varying function. Observe that, if $S^{\prime} \subset S$ and $T^{\prime} \subset T$, then $+\infty>A_{S^{\prime}, T^{\prime}}(t) \geq A_{S, T}(t)$ and, by the definition of $\eta$, we have $A_{S, T}(t)=1$ or $A_{S, T}(t) \rightarrow 0$ as $t \rightarrow \infty$, for all $S \subset I_{1}$ and $T \subset I_{2}$. Therefore,

$$
P\left(M\left(I_{1}\right)>1-1 / t, M\left(I_{2}\right)>1-1 / t\right) \sim t^{-1 / \eta} L_{\eta}(t) .
$$

Moreover, considering $\eta=\eta_{S_{0}, T_{0}}$ for some $S_{0} \subset I_{1}, T_{0} \subset I_{2}$, and so $A_{S_{0}, T_{0}}(t)=1 \leq A_{\{i\},\{j\}}(t)$, $\forall i \in S_{0}, j \in T_{0}$, we must have $A_{\{i\},\{j\}}(t)=1, \forall i \in S_{0}, j \in T_{0}$. Then $\eta=\eta_{\{i\},\{j\}}, \forall i \in S_{0}, j \in T_{0}$ and $\eta \leq \max _{i \in I_{1}, j \in I_{2}} \eta_{\{i\},\{j\}}$. But, by (28), $\eta \geq \max _{i \in I_{1}, j \in I_{2}} \eta_{\{i\},\{j\}}$ which leads to the result.

In the following we present some examples where tail independence takes place. 
Example 2.3. Consider $\left\{V_{n}\right\}_{n \geq 1}$ an i.i.d. sequence of r.v.'s with distribution $\mathrm{U}(0,1)$ and $\mathbf{X}=$ $\left(X_{1}, X_{2}, X_{3}, X_{4}\right)$ a random vector such that, $X_{1}=\min \left(V_{3}, V_{2}, V_{1}\right), X_{2}=\min \left(V_{4}, V_{2}, V_{1}\right), X_{3}=$ $\min \left(V_{4}, V_{3}, V_{1}\right)$ and $X_{4}=V_{5}$. Observe that, for $0 \leq x \leq 1, F_{X_{1}}(x)=1-(1-x)^{3}=F_{X_{2}}(x)=$ $F_{X_{3}}(x)$ and $F_{X_{4}}(x)=x$ and hence $F_{X_{1}}^{-1}(x)=1-(1-x)^{1 / 3}=F_{X_{2}}^{-1}(x)=F_{X_{3}}^{-1}(x)$ and $F_{X_{4}}^{-1}(x)=x$. Consider $I_{1}=\{1,2\}$ and $I_{2}=\{3,4\}$.

We have successively,

$$
P\left(F_{1}\left(X_{1}\right)>1-t^{-1}, F_{3}\left(X_{3}\right)>1-t^{-1}\right)=P\left(F_{2}\left(X_{2}\right)>1-t^{-1}, F_{3}\left(X_{3}\right)>1-t^{-1}\right)=t^{-4 / 3},
$$

and

$$
P\left(F_{1}\left(X_{1}\right)>1-t^{-1}, F_{4}\left(X_{4}\right)>1-t^{-1}\right)=P\left(F_{2}\left(X_{2}\right)>1-t^{-1}, F_{4}\left(X_{4}\right)>1-t^{-1}\right)=t^{-2} .
$$

Hence, by Proposition 2.4 we must derive $\eta_{(\{1,2\},\{3,4\})}=3 / 4$.

In fact, applying (27), after some calculations we have

$$
\begin{aligned}
& P\left(M\left(I_{1}\right)>1-t^{-1} x, M\left(I_{2}\right)>1-t^{-1} y\right) \\
= & \begin{cases}2 t^{-4 / 3} x y^{1 / 3}+2 t^{-2} x y-t^{-4 / 3} x^{4 / 3}-2 t^{-7 / 3} x y^{4 / 3}-2 t^{-7 / 3} x^{4 / 3} y & , x \leq y \\
t^{-4 / 3} y x^{1 / 3}+2 t^{-2} x y-3 t^{-7 / 3} x^{1 / 3} y^{2}-t^{-7 / 3} x^{4 / 3} y & , x>y .\end{cases}
\end{aligned}
$$

According to (24), coefficient $\eta_{\left(I_{1}, I_{2}\right)}$ can be obtained by taking $x=y=1$ in the expression above, and by (20) we obtain

$$
c_{(\{1,2\},\{3,4\})}(x, y)= \begin{cases}2 x y^{1 / 3}-x^{4 / 3} & , x \leq y \\ y x^{1 / 3} & , x>y .\end{cases}
$$

which is homogeneous of order $4 / 3$.

Similarly, if we consider $I_{1}=\{1,2,3\}$ and $I_{2}=\{4\}$ we obtain $\eta_{(\{1,2,3\},\{4\})}=1 / 2$ and $c_{(\{1,2,3\},\{4\})}(x, y)=x y$, and if $I_{1}=\{1\}$ and $I_{2}=\{2,3,4\}$ we have $\eta_{(\{1\},\{2,3,4\})}=3 / 4$ and

$$
c_{(\{1\},\{2,3,4\})}(x, y)=\left\{\begin{array}{ll}
x y^{1 / 3} & , x \leq y \\
2 y x^{1 / 3}-y^{4 / 3} & , x>y
\end{array}=c_{(\{1,2\},\{3,4\})}(y, x) .\right.
$$

Example 2.4. Consider $\mathbf{X}=\left(X_{1}, \ldots X_{d}\right)$ a standard d-variate Gaussian random vector with positive definite correlation matrix. The bivariate tail-dependence structure is given by

$$
P\left(F_{i}\left(X_{i}\right)>1-1 / t, F_{j}\left(X_{j}\right)>1-1 / t\right) \sim C_{\rho_{i, j}} t^{-2 /\left(1+\rho_{i, j}\right)}(\log (t))^{-\rho_{i, j} /\left(1+\rho_{i, j}\right)}, \text { as } t \rightarrow \infty,
$$

for $i, j \in\{1, \ldots, d\}, i<j$, where $\rho_{i, j}=\operatorname{corr}\left(X_{i}, X_{j}\right) \notin\{-1,1\}$ and

$$
C_{\rho_{i, j}}=\left(1+\rho_{i, j}\right)^{3 / 2}\left(1-\rho_{i, j}\right)^{-1 / 2}(4 \pi)^{-\rho_{i, j} /\left(1+\rho_{i, j}\right)} .
$$

Hence [25) holds for $I=\{i\}$ and $J=\{j\}$ with $\eta_{i, j}=\left(1+\rho_{i, j}\right) / 2$ (see Ledford and Tawn [12], 1996; Draisma et al. [3], 2004). According to Hua and Joe ([8], 2011), 25] also holds for non-empty sets $I_{1}, I_{2} \subset\{1, \ldots, d\}$. If we consider $\rho_{\left(I_{1}, I_{2}\right)}=\max \left\{\rho_{i, j}: i \in I_{1}, j \in I_{2}\right\}$ then, by Proposition 2.4. we find $\eta_{\left(I_{1}, I_{2}\right)}=\left(1+\rho_{\left(I_{1}, I_{2}\right)}\right) / 2$, provided the left-hand side of 24) is non-null. 


\section{Estimation}

Several estimators for the bivariate stable tail dependence function in (17) or even for the more general $d$-variate stable tail dependence function

$$
\lim _{t \rightarrow \infty} t P\left(F_{1}\left(X_{1}\right)>1-\frac{x_{1}}{t} \vee \ldots \vee F_{d}\left(X_{d}\right)>1-\frac{x_{d}}{t}\right)
$$

have been considered in literature. For a survey, see Krajina (2010) [11]. According to relation (18), they can be applied to our function $l^{\left(I_{1}, I_{2}\right)}\left(x^{-1}, y^{-1}\right)$.

We remark that these are based on asymptotic results that depend on a sequence of positive integers, $\left\{k_{n}\right\}$, going to infinity at a lower rate than $n$. For instance, the estimator based on (17) by plugging-in the respective empirical counterparts given by

$$
\frac{n}{k_{n}} P_{n}\left(\widehat{F}_{1}\left(X_{1}\right)>1-\frac{k_{n}}{n} x \vee \widehat{F}_{2}\left(X_{2}\right)>1-\frac{k_{n}}{n} y\right)=\frac{1}{k_{n}} \sum_{i=1}^{n} \mathbf{1}_{\left\{\widehat{F}_{1}\left(X_{1}\right)>1-\frac{k_{n}}{n} x \vee \widehat{F}_{2}\left(X_{2}\right)>1-\frac{k_{n}}{n} y\right\}},
$$

where $\widehat{F}_{l}(u)=n^{-1} \sum_{k=1}^{n} \mathbf{1}_{\left\{X_{k} \leq u\right\}}$ is the empirical d.f. of $F_{l}, l=1,2$, is consistent and asymptotically normal if $\left\{k_{n}\right\}$ is an intermediate sequence, i.e., $k_{n} \rightarrow \infty$ and $k_{n} / n \rightarrow 0$, as $n \rightarrow \infty$ (Huang 1992 [9]). The choose of the value $k$ in the sequence $\left\{k_{n}\right\}$ that allows the better trade-off between bias and variance is of major difficulty, since small values of $k$ come along with a large variance whenever an increasing $k$ results in a strong bias. Therefore, simulation studies have been carried out in order to find the best value of $k$ that allows this compromise.

As mentioned before, the upper-tail dependence function in (14) can be viewed as an extension of the bivariate upper-tail dependence function of Schmidt and Stadtmüller (2006) given in (19), by taking in this limit the random pair $\left(M\left(I_{1}\right), M\left(I_{2}\right)\right)$ instead of $\left(F\left(X_{1}\right), F\left(X_{2}\right)\right)$. The estimators considered in Schmidt and Stadtmüller (2006), for which strong consistency and asymptotic normality have been established, allow to estimate our function $\Lambda_{U}^{\left(I_{1}, I_{2}\right)}(x, y)$, as well as coefficient $\epsilon_{\left(I_{1}, I_{2}\right)}=\Lambda_{U}^{\left(I_{1}, I_{2}\right)}(1,1)$. However they are also based on asymptotic results with the same drawback of including an intermediate sequence, already referred above.

In order to overcome this problem, we shall present a totally different and very simple approach. More precisely, the following result suggests an estimation procedure for the $d$-variate stable tail dependence function in (31) that only evolves a sample mean.

Proposition 3.1. Under the conditions of Proposition 2.1, we have, for $l\left(x_{1}, \ldots, x_{d}\right)=-\log F\left(x_{1}, \ldots, x_{d}\right)$,

$$
l\left(x_{1}, \ldots, x_{d}\right)=\frac{E\left(F_{1}\left(X_{1}\right)^{x_{1}} \vee \ldots \vee F_{d}\left(X_{d}\right)^{x_{d}}\right)}{1-E\left(F_{1}\left(X_{1}\right)^{x_{1}} \vee \ldots \vee F_{d}\left(X_{d}\right)^{x_{d}}\right)}
$$

Dem. Consider for $G(x)=\exp (-1 / x)$. Observe that

$$
E\left(G\left(x_{1} X_{1}\right) \vee \ldots \vee G\left(x_{d} X_{d}\right)\right)=E\left(G\left(x_{1} X_{1} \vee \ldots \vee x_{d} X_{d}\right)\right)
$$

and the d.f. of $x_{1} X_{1} \vee \ldots \vee x_{d} X_{d}$ is given by

$$
\begin{aligned}
P\left(x_{1} X_{1} \vee \ldots \vee x_{d} X_{d} \leq u\right) & =P\left(X_{1} \leq u / x_{1}, \ldots, X_{d} \leq u / x_{d}\right) \\
& =F\left(u x_{1}^{-1}, \ldots, u x_{d}^{-1}\right) \\
& =\exp \left(-l\left(u x_{1}^{-1}, \ldots, u x_{d}^{-1}\right)\right) .
\end{aligned}
$$


Hence

$$
\begin{aligned}
& E\left(G\left(x_{1} X_{1} \vee \ldots \vee x_{d} X_{d}\right)\right) \\
= & \int_{0}^{\infty} \exp \left(-u^{-1}\right) \exp \left(-l\left(u x_{1}^{-1}, \ldots, u x_{d}^{-1}\right)\right) \frac{d}{d u}\left(-l\left(u x_{1}^{-1}, \ldots, u x_{d}^{-1}\right)\right) \\
= & \int_{0}^{\infty} \exp \left(-u^{-1}\right) \exp \left(-u^{-1} l\left(x_{1}^{-1}, \ldots, x_{d}^{-1}\right)\right) \frac{d}{d u}\left(-u^{-1} l\left(x_{1}^{-1}, \ldots, x_{d}^{-1}\right)\right) \\
= & l\left(x_{1}^{-1}, \ldots, x_{d}^{-1}\right) \int_{0}^{\infty} \exp \left(-u^{-1}\left(1+l\left(x_{1}^{-1}, \ldots, x_{d}^{-1}\right)\right)\right) u^{-2} d u \\
= & \frac{l\left(x_{1}^{-1}, \ldots, x_{d}^{-1}\right)}{1+l\left(x_{1}^{-1}, \ldots, x_{d}^{-1}\right)} .
\end{aligned}
$$

Now just observe that $G\left(x_{1} X_{1} \vee \ldots \vee x_{d} X_{d}\right) \stackrel{d}{=} F_{1}\left(X_{1}\right)^{1 / x_{1}} \vee \ldots \vee F_{d}\left(X_{d}\right)^{1 / x_{d}}$

Remark 3.1. Observe that the d-variate stable tail dependence function in (31) corresponds to $-\log F\left(x_{1}^{-1}, \ldots, x_{d}^{-1}\right)$.

By applying Proposition 3.1 with $x_{j}$ replaced by $x_{j}^{-1}, j=1, \ldots, d$, we get the following corollary.

Corollary 3.2. Under the conditions of Proposition 2.1, we have

$$
\begin{gathered}
x \epsilon_{I_{1}} \equiv l^{\left(I_{1}, \emptyset\right)}\left(x^{-1}, x^{-1}\right)=\frac{E\left(M\left(I_{1}\right)^{1 / x}\right)}{1-E\left(M\left(I_{1}\right)^{1 / x}\right)}, \\
y \epsilon_{I_{2}} \equiv l^{\left(\emptyset, I_{2}\right)}\left(y^{-1}, y^{-1}\right)=\frac{E\left(M\left(I_{2}\right)^{1 / y}\right)}{1-E\left(M\left(I_{2}\right)^{1 / y}\right)}
\end{gathered}
$$

and

$$
l^{\left(I_{1}, I_{2}\right)}\left(x^{-1}, y^{-1}\right)=\frac{E\left(M\left(I_{1}\right)^{1 / x} \vee M\left(I_{2}\right)^{1 / y}\right)}{1-E\left(M\left(I_{1}\right)^{1 / x} \vee M\left(I_{2}\right)^{1 / y}\right)}
$$

Consider the estimators derived from Proposition 2.1 and Corollary 3.2 by plugging-in the respective sample means, respectively,

$$
\widetilde{l}\left(x_{1}, \ldots, x_{d}\right)=\frac{\overline{F_{1}\left(X_{1}\right)^{x_{1}} \vee \ldots \vee F_{d}\left(X_{d}\right)^{x_{d}}}}{1-\overline{F_{1}\left(X_{1}\right)^{x_{1}} \vee \ldots \vee F_{d}\left(X_{d}\right)^{x_{d}}}}
$$

and

$$
x \widetilde{\epsilon}_{I_{1}}=\frac{\overline{M\left(I_{1}\right)^{1 / x}}}{1-\overline{M\left(I_{1}\right)^{1 / x}}}, y \widetilde{\epsilon}_{I_{2}}=\frac{\overline{M\left(I_{2}\right)^{1 / y}}}{1-\overline{M\left(I_{2}\right)^{1 / y}}} \text { and } \widetilde{l}^{\left(I_{1}, I_{2}\right)}\left(x^{-1}, y^{-1}\right)=\frac{\overline{M\left(I_{1}\right)^{1 / x} \vee M\left(I_{2}\right)^{1 / y}}}{1-\overline{M\left(I_{1}\right)^{1 / x} \vee M\left(I_{2}\right)^{1 / y}}}
$$

where

$$
\overline{M\left(I_{1}\right)^{1 / x}}=\frac{1}{n} \sum_{i=1}^{n} \bigvee_{j \in I_{1}} F_{j}\left(X_{j}^{(i)}\right)^{1 / x}, \quad \overline{M\left(I_{2}\right)^{1 / y}}=\frac{1}{n} \sum_{i=1}^{n} \bigvee_{j \in I_{2}} F_{j}\left(X_{j}^{(i)}\right)^{1 / y}
$$


and

$$
\overline{M\left(I_{1}\right)^{1 / x} \vee M\left(I_{2}\right)^{1 / y}}=\frac{1}{n} \sum_{i=1}^{n}\left(\bigvee_{j \in I_{1}} F_{j}\left(X_{j}^{(i)}\right)^{1 / x} \vee \bigvee_{j \in I_{2}} F_{j}\left(X_{j}^{(i)}\right)^{1 / y}\right) .
$$

We will consider two situations: the first one for known margins and the second one for unknown margins.

In case the margins are known, they become unit Fréchet by transformation $-1 / \log F_{j}\left(X_{j}\right)$ for $j \in I \subset\{1, \ldots, d\}$.

It is quite straightforward to deduce the consistency and asymptotic normality of estimators (37) and (38) by the well-known Delta Method.

Proposition 3.3. Under the conditions of Proposition [2.1, we have

$$
\sqrt{n}\left(\widetilde{l}\left(x_{1}, \ldots, x_{d}\right)-l\left(x_{1}, \ldots, x_{d}\right)\right) \rightarrow N\left(0, \sigma^{2}\right),
$$

where $\widetilde{l}\left(x_{1}, \ldots, x_{d}\right)$ is the estimator derived from Proposition 3.1 by plugging-in the respective sample mean given in (37) and

$$
\sigma^{2}=\frac{l\left(x_{1}, \ldots, x_{d}\right)\left(1+l\left(x_{1}, \ldots, x_{d}\right)\right)^{2}}{\left(2+l\left(x_{1}, \ldots, x_{d}\right)\right)} .
$$

Dem. Let $Y_{i}, i=1, \ldots, n$, be independent copies of $Y=F_{1}\left(X_{1}\right)^{x_{1}} \vee \ldots \vee F_{d}\left(X_{d}\right)^{x_{d}}$. We have that $\sqrt{n}\left(\bar{Y}-\mu_{Y}\right) \rightarrow N\left(0, \sigma_{Y}^{2}\right)$, where $\mu_{Y}=E\left(F_{1}\left(X_{1}\right)^{x_{1}} \vee \ldots \vee F_{d}\left(X_{d}\right)^{x_{d}}\right)$ and $\sigma_{Y}^{2}=\operatorname{Var}\left(F_{1}\left(X_{1}\right)^{x_{1}} \vee\right.$ $\left.\ldots \vee F_{d}\left(X_{d}\right)^{x_{d}}\right)$. By a similar reasoning of (33) we derive

$$
E\left(\left(F_{1}\left(X_{1}\right)^{x_{1}} \vee \ldots \vee F_{d}\left(X_{d}\right)^{x_{d}}\right)^{2}\right)=\frac{l\left(x_{1}, \ldots, x_{d}\right)}{2+l\left(x_{1}, \ldots, x_{d}\right)}
$$

and hence,

$$
\operatorname{Var}\left(\left(F_{1}\left(X_{1}\right)^{x_{1}} \vee \ldots \vee F_{d}\left(X_{d}\right)^{x_{d}}\right)^{2}\right)=\frac{l\left(x_{1}, \ldots, x_{d}\right)}{\left(2+l\left(x_{1}, \ldots, x_{d}\right)\right)\left(1+l\left(x_{1}, \ldots, x_{d}\right)\right)^{2}}
$$

Let $g(x)=(1-x)^{-1}-1$. We have $\left[g^{\prime}\left(\mu_{Y}\right)\right]^{2}=\left(1-\mu_{Y}\right)^{-4}$ and, by the Delta Method, $\sqrt{n}(g(\bar{Y})-$ $\left.x \epsilon_{I_{1}}\right) \rightarrow N\left(0, \sigma_{Y}^{2}\left(1-\mu_{Y}\right)^{-4}\right)$.

Corollary 3.4. Under the conditions of Proposition 2.1, we have

$$
\begin{aligned}
& \sqrt{n}\left(x \widetilde{\epsilon}_{I_{1}}-x \epsilon_{I_{1}}\right) \rightarrow N\left(0, \sigma_{1}^{2}\right), \\
& \sqrt{n}\left(y \widetilde{\epsilon}_{I_{2}}-y \epsilon_{I_{2}}\right) \rightarrow N\left(0, \sigma_{2}^{2}\right)
\end{aligned}
$$

and

$$
\sqrt{n}\left(\widetilde{l}^{\left(I_{1}, I_{2}\right)}\left(x^{-1}, y^{-1}\right)-l^{\left(I_{1}, I_{2}\right)}\left(x^{-1}, y^{-1}\right)\right) \rightarrow N\left(0, \sigma_{3}^{2}\right),
$$

where $x \widetilde{\epsilon}_{I_{1}}, y \widetilde{\epsilon}_{I_{2}}$ and $\widetilde{l}^{\left(I_{1}, I_{2}\right)}\left(x^{-1}, y^{-1}\right)$ are given in (38) and

$$
\begin{gathered}
\sigma_{1}^{2}=\frac{x \epsilon_{I_{1}}\left(1+x \epsilon_{I_{1}}\right)^{2}}{\left(2+x \epsilon_{I_{1}}\right)}, \\
\sigma_{2}^{2}=\frac{y \epsilon_{I_{2}}\left(1+y \epsilon_{I_{2}}\right)^{2}}{\left(2+y \epsilon_{I_{2}}\right)}
\end{gathered}
$$

and

$$
\sigma_{3}^{2}=\frac{l^{\left(I_{1}, I_{2}\right)}\left(x^{-1}, y^{-1}\right)\left(1+l^{\left(I_{1}, I_{2}\right)}\left(x^{-1}, y^{-1}\right)\right)^{2}}{\left(2+l^{\left(I_{1}, I_{2}\right)}\left(x^{-1}, y^{-1}\right)\right)}
$$


Based on the definition in (14), a natural estimator for the upper-tail dependence function is

$$
\widetilde{\Lambda}_{U}^{\left(I_{1}, I_{2}\right)}(x, y)=x \widetilde{\epsilon_{I_{1}}}+y \widetilde{\epsilon_{I_{2}}}-\widetilde{l}^{\left(I_{1}, I_{2}\right)}\left(x^{-1}, y^{-1}\right),
$$

with $x \widetilde{\epsilon_{1}}, y \widetilde{\epsilon_{I_{2}}}$ and $\widetilde{l}^{\left(I_{1}, I_{2}\right)}\left(x^{-1}, y^{-1}\right)$ stated in (38). Hence we have the following estimator for the extremal coefficient of dependence between $\mathbf{X}_{I_{1}}$ and $\mathbf{X}_{I_{2}}$ :

$$
\widetilde{\epsilon}_{\left(I_{1}, I_{2}\right)}=\widetilde{\epsilon_{I_{1}}}+\widetilde{\epsilon_{I_{2}}}-\widetilde{\epsilon}_{I_{1} \cup I_{2}}
$$

where $\widetilde{\epsilon}_{I_{1} \cup I_{2}}=\widetilde{l}^{\left(I_{1}, I_{2}\right)}(1,1)$.

Proposition 3.5. Estimators $\widetilde{l}\left(x_{1}, \ldots, x_{d}\right)$ and $\widetilde{\Lambda}_{U}^{\left(I_{1}, I_{2}\right)}(x, y)$ in (37) and 48), respectively, are strong consistent. Consequently, the same holds for $\tilde{\epsilon}_{\left(I_{1}, I_{2}\right)}$ in 49 .).

Dem. Just observe that, as the sample mean $\overline{M\left(I_{1}\right)^{1 / x}}$ converges almost surely to the mean value $E\left(M\left(I_{1}\right)^{1 / x}\right)$, i.e., $\overline{M\left(I_{1}\right)^{1 / x}} \stackrel{a . s}{\longrightarrow} E\left(M\left(I_{1}\right)^{1 / x}\right)$, then $x \widetilde{\epsilon_{I_{1}}}=g\left(\overline{M\left(I_{1}\right)^{1 / x}}\right) \stackrel{a . s}{\longrightarrow} x \epsilon_{I_{1}}=$ $g\left(E\left(M\left(I_{1}\right)^{1 / x}\right)\right)$, where $g(x)=(1-x)^{-1}-1$. Analogously for $y \widetilde{\epsilon_{I_{2}}}, \widetilde{l}^{\left(I_{1}, I_{2}\right)}\left(x^{-1}, y^{-1}\right)$ and $\widetilde{l}\left(x_{1}, \ldots, x_{d}\right)$. Now, the strong consistency of $\widetilde{\Lambda}_{U}^{\left(I_{1}, I_{2}\right)}(x, y)$ is straightforward from

$$
\left|\widetilde{\Lambda}_{U}^{\left(I_{1}, I_{2}\right)}(x, y)-\Lambda_{U}^{\left(I_{1}, I_{2}\right)}(x, y)\right| \leq\left|x \widetilde{\epsilon_{I_{1}}}-x \epsilon_{I_{1}}\right|+\left|y \widetilde{\epsilon_{I_{2}}}-y \epsilon_{I_{2}}\right|+\left|\widetilde{l}^{\left(I_{1}, I_{2}\right)}\left(x^{-1}, y^{-1}\right)-l^{\left(I_{1}, I_{2}\right)}\left(x^{-1}, y^{-1}\right)\right| .
$$

Now consider $\widehat{F}_{j}$ the empirical d.f. of $F_{j}, j=1, \ldots, d$,

$$
\widehat{F}_{j}(u)=\frac{1}{n+1} \sum_{k=1}^{n} \mathbf{1}_{\left\{X_{j}^{(k)} \leq u\right\}} .
$$

The denominator $n+1$ instead of $n$ in the empirical d.f. concerns estimation accuracy and other modifications can be used. For a discussion see, for instance, Beirlant et al. 11 (2004).

In case of unknown margins, we can replace $F_{j}$ by the respective empirical d.f. $\widehat{F}_{j}, j=1, \ldots, d$, in (37) and (38). More precisely, we have

$$
\widehat{l}\left(x_{1}, \ldots, x_{d}\right)=\frac{\overline{\widehat{F}_{1}\left(X_{1}\right)^{x_{1}} \vee \ldots \vee \widehat{F}_{d}\left(X_{d}\right)^{x_{d}}}}{1-\overline{\widehat{F}_{1}\left(X_{1}\right)^{x_{1}} \vee \ldots \vee \widehat{F}_{d}\left(X_{d}\right)^{x_{d}}}},
$$

as well as,

$$
x \widehat{\epsilon}_{I_{1}}=\frac{\overline{\widehat{M}\left(I_{1}\right)^{1 / x}}}{1-\widehat{\widehat{M}\left(I_{1}\right)^{1 / x}}}, y \widehat{\epsilon}_{I_{2}}=\frac{\widehat{\widehat{M}\left(I_{2}\right)^{1 / y}}}{1-\widehat{\widehat{M}\left(I_{2}\right)^{1 / y}}} \text { and } \widehat{l}^{\left(I_{1}, I_{2}\right)}\left(x^{-1}, y^{-1}\right)=\frac{\widehat{\widehat{M}\left(I_{1}\right)^{1 / x} \vee \widehat{M}\left(I_{2}\right)^{1 / y}}}{1-\widehat{\widehat{M}\left(I_{1}\right)^{1 / x} \vee \widehat{M}\left(I_{2}\right)^{1 / y}}}
$$

where

$$
\begin{gathered}
\overline{\widehat{F}_{1}\left(X_{1}\right)^{x_{1}} \vee \ldots \vee \widehat{F}_{d}\left(X_{d}\right)^{x_{d}}}=\frac{1}{n} \sum_{i=1}^{n} \bigvee_{j \in\{1, \ldots, d\}} \widehat{F}_{j}\left(X_{j}^{(i)}\right)^{x_{j}}, \\
\overline{\widehat{M}\left(I_{1}\right)^{1 / x}}=\frac{1}{n} \sum_{i=1}^{n} \bigvee_{j \in I_{1}} \widehat{F}_{j}\left(X_{j}^{(i)}\right)^{1 / x}, \quad \overline{\widehat{M}\left(I_{2}\right)^{1 / y}}=\frac{1}{n} \sum_{i=1}^{n} \bigvee_{j \in I_{2}} \widehat{F}_{j}\left(X_{j}^{(i)}\right)^{1 / y}
\end{gathered}
$$

and

$$
\widehat{\widehat{M}\left(I_{1}\right)^{1 / x} \vee \widehat{M}\left(I_{2}\right)^{1 / y}}=\frac{1}{n} \sum_{i=1}^{n}\left(\bigvee_{j \in I_{1}} \widehat{F}_{j}\left(X_{j}^{(i)}\right)^{1 / x} \vee \bigvee_{j \in I_{2}} \widehat{F}_{j}\left(X_{j}^{(i)}\right)^{1 / y}\right) .
$$

We still have asymptotic normality of estimators in (52)-(54) from the following result stated in Fermanian et al. (2002, [6], Theorem 6). 
Theorem 3.6. (Fermanian et al. (2002) [6], Theorem 6) Let $F$ have continuous marginals and let copula $C_{F}$ in (2) have continuous partial derivatives. Then

$\frac{1}{\sqrt{n}} \sum_{i=1}^{n}\left\{J\left(\widehat{F}_{1}\left(X_{1}^{(i)}\right), \ldots, \widehat{F}_{d}\left(X_{d}^{(i)}\right)\right)-E\left(J\left(F_{1}\left(X_{1}^{(i)}\right), \ldots, F_{d}\left(X_{d}^{(i)}\right)\right)\right)\right\} \rightarrow \int_{[0,1]^{d}} \mathbb{G}\left(u_{1}, \ldots, u_{d}\right) d J\left(u_{1}, \ldots, u_{d}\right)$

in distribution in $\ell^{\infty}\left([0,1]^{d}\right)$, where the limiting process and $\mathbb{G}$ are centered Gaussian, and $J$ : $[0,1]^{d} \rightarrow \mathbb{R}$ is of bounded variation, continuous from above and with discontinuities of the first kind (Neuhaus, 1971 [20]).

The asymptotic normality of estimators (50) and (51) is now derived from a general version of the Delta Method as considered in Schmidt and Stadtmüller [22] (2006; Theorem 13).

We also state strong consistency of estimators $\widehat{l}\left(x_{1}, \ldots, x_{d}\right)$ in (50) and

$$
\widehat{\Lambda}_{U}^{\left(I_{1}, I_{2}\right)}(x, y)=x \widehat{\epsilon_{I_{1}}}+y \widehat{\epsilon_{I_{2}}}-\widehat{l}^{\left(I_{1}, I_{2}\right)}\left(x^{-1}, y^{-1}\right),
$$

with $x \widehat{\epsilon_{1}}, y \widehat{\epsilon_{I_{2}}}$ and $\widehat{l}^{\left(I_{1}, I_{2}\right)}\left(x^{-1}, y^{-1}\right)$ given in (51), and hence of estimator

$$
\widehat{\epsilon}_{\left(I_{1}, I_{2}\right)}=\widehat{\epsilon_{I_{1}}}+\widehat{\epsilon_{I_{2}}}-\widehat{\epsilon}_{I_{1} \cup I_{2}}
$$

where $\widehat{\epsilon}_{I_{1} \cup I_{2}}=\widehat{l}^{\left(I_{1}, I_{2}\right)}(1,1)$.

Proposition 3.7. Estimators $\widehat{l}\left(x_{1}, \ldots, x_{d}\right)$ in (50) and $\widehat{\Lambda}_{U}^{\left(I_{1}, I_{2}\right)}(x, y)$ in (55) are strong consistent. Therefore, the same holds for estimator $\widehat{\epsilon}_{\left(I_{1}, I_{2}\right)}$ in (56)).

Dem. The proof runs along the same lines as the one of Proposition 3.5. We only prove the more general case $\widehat{l}\left(x_{1}, \ldots, x_{d}\right) \stackrel{a . s}{\longrightarrow} l\left(x_{1}, \ldots, x_{d}\right)$. Observe that

$$
\begin{aligned}
& \left|\frac{1}{n} \sum_{i=1}^{n} \bigvee_{j \in\{1, \ldots, d\}} \widehat{F_{j}}\left(X_{j}^{(i)}\right)^{x_{j}}-E\left(\bigvee_{j \in\{1, \ldots, d\}} F_{j}\left(X_{j}\right)^{x_{j}}\right)\right| \\
\leq & \left|\frac{1}{n} \sum_{i=1}^{n} \bigvee_{j \in\{1, \ldots, d\}} \widehat{F_{j}}\left(X_{j}^{(i)}\right)^{x_{j}}-\frac{1}{n} \sum_{i=1}^{n} \bigvee_{j \in\{1, \ldots, d\}} F_{j}\left(X_{j}^{(i)}\right)^{x_{j}}\right| \\
& +\left|\frac{1}{n} \sum_{i=1}^{n} \bigvee_{j \in\{1, \ldots, d\}} F_{j}\left(X_{j}^{(i)}\right)^{x_{j}}-E\left(\bigvee_{j \in\{1, \ldots, d\}} F_{j}\left(X_{j}\right)^{x_{j}}\right)\right|,
\end{aligned}
$$

where the second term converges almost surely to zero by the Strong Law of Large Numbers.

For the first term we have, successively,

$$
\begin{aligned}
& \left|\frac{1}{n} \sum_{i=1}^{n} \bigvee_{j \in\{1, \ldots, d\}} \widehat{F}_{j}\left(X_{j}^{(i)}\right)^{x_{j}}-\frac{1}{n} \sum_{i=1}^{n} \bigvee_{j \in\{1, \ldots, d\}} F_{j}\left(X_{j}^{(i)}\right)^{x_{j}}\right| \\
& \leq \frac{1}{n} \sum_{i=1}^{n} \bigvee_{j \in\{1, \ldots, d\}}\left|\widehat{F}_{j}\left(X_{j}^{(i)}\right)^{x_{j}}-F_{j}\left(X_{j}^{(i)}\right)^{x_{j}}\right| \\
& \leq \frac{1}{n} \sum_{i=1}^{n} \sum_{j \in\{1, \ldots, d\}}\left|\widehat{F}_{j}\left(X_{j}^{(i)}\right)^{x_{j}}-F_{j}\left(X_{j}^{(i)}\right)^{x_{j}}\right|,
\end{aligned}
$$

which converges almost surely to zero according to Gilat and Hill ([7], 1992; proof of Theorem 1.1). 


\section{Application to financial data}

In this section we show that tail dependence is present in financial data. Our analysis is based on negative log-returns of daily closing values of the stock market indexes, CAC 40 (France), FTSE100 (UK), SMI (Swiss), XDAX (German), Dow Jones (USA), Nasdaq (USA), SP500 (USA), HSI (China), Nikkei (Japan). The period covered is January 1993 to March 2004. More precisely, we consider the monthly maximums in each market and group the indexes in Europe (CAC 40, FTSE100, SMI, XDAX), USA (Dow Jones, Nasdaq) and Far East (HSI, Nikkei). The scatter plots in Figure 1 show the presence of dependence between the monthly maximums in Europe and USA, Europe and Far East, USA and Far East, respectively. We are interested in assessing the amount
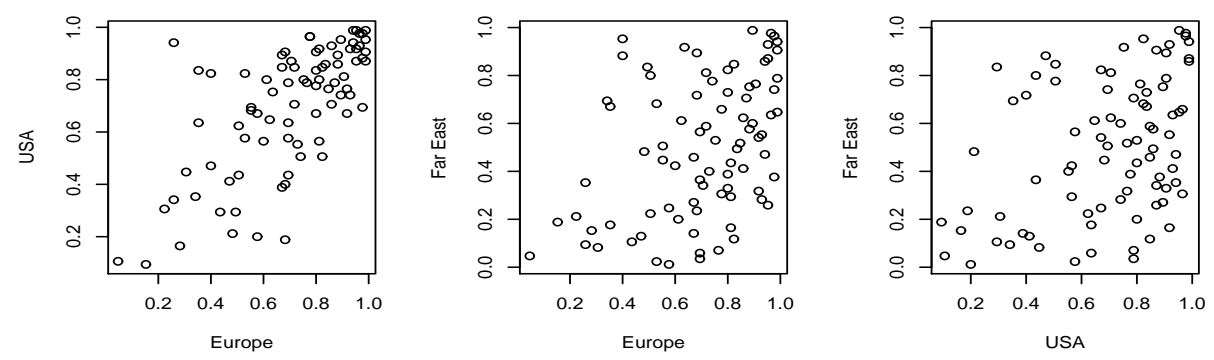

Figure 1: Scatter plots of the monthly maximums (84 data points) in Europe versus USA, Europe versus Far East and USA versus Far East.

of tail dependence between the three big world markets referred: Europe, USA and Far East, and this can be achieved through the extremal coefficient of dependence $\epsilon_{\left(I_{1}, I_{2}\right)}$, defined in (15). As we do not know the margins distribution, we use estimator $\widehat{\epsilon}_{\left(I_{1}, I_{2}\right)}$ in (56) based on ranks. In Table 1 are the obtained estimates for several groups, $I_{1}$ and $I_{2}$. One can see that the Far East market has less influence (lower values of the coefficient) but Europe and USA have a stronger effect on each other and on the respective group of foreign markets. Observe that the difference between these two magnitudes of dependence is almost in the proportion 1:2.

\begin{tabular}{|c|c|c|}
\hline$I_{1}$ & $I_{2}$ & $\widehat{\epsilon}_{\left(I_{1}, I_{2}\right)}$ \\
\hline Europe & USA & 1.008324625 \\
Europe & Far East & 0.568780467 \\
USA & Far East & 0.364358832 \\
Europe & USA $\cup$ Far East & 1.125919957 \\
USA & Europe $\cup$ Far East & 0.921498322 \\
Far East & USA $\cup$ Europe & 0.481954164 \\
\hline
\end{tabular}

Table 1: Estimates of the extremal coefficient of dependence $\widehat{\epsilon}_{\left(I_{1}, I_{2}\right)}$ for the indicated groups, $I_{1}$ and $I_{2}$.

\section{Conclusion}

In this work we introduce a new upper-tail dependence concept for a random vector which extends the one in Schmidt and Stadtmüller ([22, 2006). Our approach weakens the usual imposed multivariate tail dependence and can be treated with bivariate techniques. The new function extends the well-known relation of Huang ([9 1992) for a MEV with unit Fréchet marginals, and gives rise 
to the so-called extremal coefficient of dependence as it is expressed through the extremal coefficient in Tiago de Oliveira ([26, 1962-63) and Smith ([23, 1990). We also enlarge our discussion to tail independence in the sense of Ledford and Tawn (12, 13, 1996, 1997). At this point we are beyond MEV distributions which only admit tail dependence or (exact) independence.

In calculating the moments of the r.v.'s involved in our function, we arrive at very simple estimators whose asymptotic normality is stated. These can also be applied to the well-known stable tail dependence function. We also prove strong consistency of the proposed estimators for our measures. We end with an application to financial data presenting tail dependence.

\section{References}

[1] Beirlant, J., Goegebeur, Y., Segers, J. e Teugels, J. (2004). Statistics of Extremes: Theory and Application. John Wiley.

[2] Coles, S., Heffernan, J. and Tawn, J. (1999). Dependence measures for extreme value analysis, Extremes 2: 339-366.

[3] Draisma, G., Drees, H., Ferreira, A. and de Haan, L. (2004). Bivariate tail estimation: dependence in asymptotic independence. Bernoulli, 10, 251-280.

[4] Drees, H., and Müller, P. (2008). Fitting and validation of a bivariate model for large claims. Insurance: Mathematics and Economics 42, 638-650.

[5] Embrechts, P., Lindskog, F. and McNeil, A. (2003). Modelling Dependence with Copulas and Applications to Risk Management, In: Handbook of Heavy Tailed Distibutions in Finance, ed. S. Rachev, Elsevier, Chapter 8: 329-384.

[6] Fermanian, J.-D., Radulović, D., Wegkamp, M. (2004). Weak convergence of empirical copula processes. Bernoulli 10(5), 847-860.

[7] Gilat, D. and Hill, T. (1992) One-sided refinements of the strong law of large numbers and the Glivenko-Cantelli Theorem, Ann. Probab. 20 , 1213-1221.

[8] Hua, L., Joe, H. (2004). Tail order and intermediate tail dependence of multivariate copulas.

[9] Huang, X. (1992). Statistics of Bivariate Extreme Values. Ph. D. thesis, Tinbergen Institute Research Series 22, Erasmus University Rotterdam.

[10] Joe, H. (1997). Multivariate Models and Dependence Concepts. Chapman \& Hall, London.

[11] Krajina, A. (2010). An M-Estimator of Multivariate Tail Dependence. Tilburg: Tilburg University Press.

[12] Ledford, A. and Tawn, J. A. (1996). Statistics for near independence in multivariate extreme values. Biometrika, 83, 169-187.

[13] Ledford, A. and Tawn, J. A. (1997). Modelling Dependence within joint tail regions, J. R. Stat. Soc. Ser. B Stat. Methodol. 59, 475-499.

[14] Li, H. (2006). Tail dependence of multivariate Pareto distributions, WSU Mathematics Technical Report 2006-6. http://www.math.wsu.edu/TRS/

[15] Li, H. (2008). Tail Dependence Comparison of Survival Marshall-Olkin Copulas, Methodol. Comput. Appl. Probab., 10(1), 39-54.

[16] Li, H. (2009). Orthant tail dependence of multivariate extreme value distributions, J. Multivariate Anal., 100(1), 243-256. 
[17] Marshall, A.W., Olkin, I. (1967). A multivariate exponential distribution, J. Amer. Statist. Assoc. 62 30-44.

[18] Nelsen, R. B. (1996). Nonparametric measures of multivariate association, in Distribution with fixed marginals and related topics, IMS Lecture Notes - Monograph Series, vol. 28, 223-232.

[19] Nelsen, R.B. (2006). An Introduction to Copulas. Second Edition. Springer, New York.

[20] Neuhaus, G. (1971) On the weak convergence of stochastic processes with multidimensional time parameter. Ann. Math. Statist., 42, 1285-1295.

[21] Schmid, F., Schmidt, R. (2007). Multivariate conditional versions of Spearman's rho and related measures of tail dependence. J. Multivariate Anal., 98, 1123-1140.

[22] Schmidt, R., Stadtmüller, U. (2006). Nonparametric estimation of tail dependence, The Scandinavian Journal of Statistics 33, 307-335.

[23] Smith, R.L. (1990). Max-stable processes and spatial extremes. Preprint, Univ. North Carolina, USA.

[24] Smith, R.L., Weissman, I. (1996). Characterization and estimation of the multivariate extremal index. Manuscript, UNC.

[25] Sibuya, M. (1960). Bivariate extreme statistics. Ann. Inst. Statist. Math. 11, 195-210.

[26] Tiago de Oliveira, J. (1962/63). Structure theory of bivariate extremes, extensions. Est. Mat., Estat. e Econ. 7, 165-195.

[27] Wolff, E. F. (1980). N-dimensional measures of dependence. Stochastica 4 (3), 175-188. 